EAPS8-1210 Association between type 1 diabetes and celiac

\title{
disease in children
}

Kmiha $\mathbf{S}^{*}$, Aloulou $\mathrm{H}^{*}$, Amous $\mathbf{M}^{*}$, Maaloul I*, Kamoun $\mathrm{Th}^{*}$, Hachicha $\mathrm{H}^{* *}$, Chabchoub I*, Masmoudi $\mathrm{H}^{* *}$, Hachicha $\mathrm{M}^{*}$

* Department of Pediatrics, Hedi Chaker University Hospital of Sfax, TUNISIA ** Laboratory of immunology, Habib Bourguiba University Hospital of Sfax, TUNISIA

- Background: The diagnosis of atypical and silent forms of celiac disease (CD) is important in view of the risk of serious complications such as malabsorption and digestive cancers. The increase in the incidence of ce disease in diabetic children has been reported in many studies.

Aim : to determine the prevalence of $\mathrm{CD}$ among diabetic children and to study its peculiarities in children with diabetes.

\section{Patients and methods: A}

prospective study was conducted among 463 children who were hospitalized or followed for type 1 diabetes at oncet over a 16-year period (2000-2015) at the

Pediatric Department of Hedi Chaker University Hospital of Sfax, Tunisia. A systematic screening for antigliadin antibodies (AGA), antiendomysium antibodies (AEA) and anti-transglutaminase antibodies (ATGA) was performed. Jejunal biopsy was made whenever serology was positive.

Results: AGA were positive in 30 children, AEA were positive in 10 children and ATGA

were positive in 14 children. Jejunal biopsy was performed in 12 cases, it was normal in 2 cases and showed histological findings of celiac disease in 10 cases (prevalence: $2.15 \%$ ).
Patients with diabetes and celiac disease were divided into 7 girls and 3 boys, the mean age at diagnosis was 6 years old ( 3 to 10 years old). Celiac disease was diagnosed 2 years after diabetes in 2 cases. Chronic diarrhea was noted in only one case and growth retardation was noted in 2 cases. In other cases, the CD was silent. Regarding the control of diabetes, the average glycated hemoglobin was $10 \%$. Four children were put on gluten-free diet.

Conclusion : the prevalence of CD in diabetic children is similar to that found in the literature series, which varies between 0.9 and $16.4 \%$. This frequency is probably underestimated because not all patients with positive serology had a jejunal biopsy. Patients diagnosed with routine screening are often asymptomatic and should be informed about the diagnosis andtreatment to prevent further complications. 